\title{
HE Neutrinos beyond Standard Model: steriles and secret interactions
}

\author{
Ninetta Saviano ${ }^{a}$ \\ ${ }^{a}$ INFN, sezione di Napoli, Complesso Univ. Monte S. Angelo, I-80126 Napoli, Italy \\ E-mail: nsaviano@na.infn.it
}

We discuss possible observable features in high and ultrahigh energy neutrino fluxes due to active-sterile secret interactions mediated by a new pseudoscalar particle. Active-sterile neutrino interactions become relevant at very different energy scales depending on the masses of the scalar mediator and of sterile neutrino. As consequence, the final active fluxes can present a measurable depletion observable in current and future future experiments. We adopted two prescriptions for the neutrino flux, namely high-energy represented by a power-law, and ultra high-energy with cosmological origin (cosmogenic) in order to take into account the multi-scale energy phenomenology, varying from $10^{6} \mathrm{GeV}$ up to $10^{9} \mathrm{GeV}$. When the depletion is around $10^{6} \mathrm{GeV}$, this effect could be very interesting for IceCube because it can produce a cutoff-like feature in the spectrum, which could potentially explain the lack of observed events above $10 \mathrm{PeV}$. For larger values of mediator and sterile masses, the depletion effect instead could be only visible at larger energy, around $10^{9} \mathrm{GeV}$, with future experiments like GRAND.

$37^{\text {th }}$ International Cosmic Ray Conference (ICRC 2021)

July 12 th - 23rd, 2021

Online - Berlin, Germany 


\section{Introduction}

Tested and verified with extremely high precision, the Standard Model of particle physics is an elegant way of describing the relationships between particles and their interactions. However, the neutrino sector still represents a partially un-known territory. Fundamental questions like the nature of neutrinos (Dirac or Majorana) or the possible connection of their small masses with physics Beyond the Standard Model (BSM) can represent possible windows on new physics. One of the simplest additions would be extra neutrinos with a huge mass. These extra neutrino species are named sterile in the sense they are singlets for the gauge group since they do not standard interact with the other particles, except for gravity. Their masses are usually postulated heavy, but lighter sterile neutrinos are anyway possible. Another possible extension of the standard model is represented by new kind of interactions, usually referred as secret interactions (SI), involving both active and sterile neutrinos. We can use different environments and so different information to test and verify sterile neutrinos and secret interactions. A the same time we could have the unique opportunity trough these new messengers to decipher the mysteries of the Universe and astrophysical sources. Limits on neutrino SI can be set by observations of laboratory processes. Even if the neutrinos are not detected directly, their presence can be inferred by precise measurements of other particles. Among them very important are laboratory constraints come from meson decays. A very promising opportunity to test secret interactions and also sterile neutrinos is represented by neutrinos from astrophysical sources that travel a long distance through the Cosmic Neutrino Background (CNB). Finally, an important arena to test these exotic scenario is the Early Universe, being density to different neutrino properties at different epochs [3-6]. In this work we present possible observable features in high ( below $100 \mathrm{PeV}$ ) and ultrahigh energy (comsogenic) neutrino fluxes due to active-sterile secret interactions mediated by a new pseudoscalar particle, that can be measurable in current and future experiments $[1,2]$.

\section{THE MODEL}

Our idea is a a particular scheme of SI, where the new interaction, mediated by a new pseudoscalar boson, involves both active and sterile neutrinos. Schematically, extremely energetic active neutrinos, during their path to Earth, can collide with a relic neutrino of the CNB producing a flux of sterile neutrinos. As a consequence, the final active fluxes can show a measurable depletion, called absorption, possibly observable in present and future experiments, depending on the energy of colliding neutrino. Assuming that neutrinos are described by Majorana spinors and considering just one sterile neutrino $v_{s}$ coupling with the active ones, and considering negligible active-sterile mixing, the new interaction involving active and sterile neutrinos is given by

$$
\mathcal{L}_{\mathrm{SI}}=\sum_{\alpha} \lambda_{\alpha} \bar{v}_{\alpha} \gamma_{5} v_{s} \varphi
$$

where $\alpha=e, \mu, \tau$ and $\lambda_{\alpha}$ are dimensionless free couplings, the free parameters of the model, providing an ample freedom of choice for our model. In our investigation we therefore consider two benchmark cases: the most natural possibility is $\lambda_{e}=\lambda_{\mu}=\lambda_{\tau}$, since it preserves lepton universality. However, also the case in which only $\lambda_{\tau} \neq 0$ is very interesting since it will result only 


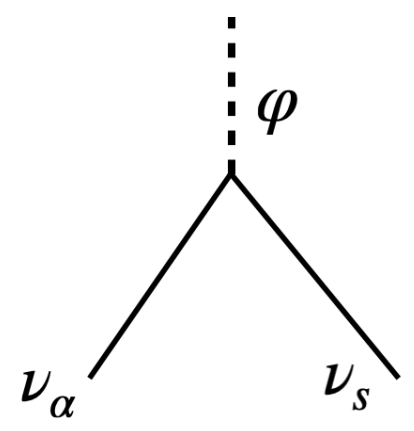

Figure 1: Diagram for the secret interaction of active and sterile neutrinos mediated by a new pseudoscalar boson.

very weakly constrained by mesons decay leading therefore to larger effects on the astrophysical fluxes.

As mentioned in [1], the cross section for the active-sterile collisions shows a resonance in the t-channel: a sterile neutrino with momentum $p$, colliding with a fixed active neutrino, can decay producing an active neutrino and a scalar mediator which is then exchanged with the active neutrino. The resonance condition $t=M_{\varphi}^{2}$, for the energy of the final sterile and of active neutrino, reads:

$$
E_{s}^{i}=\frac{m_{i}^{2}+m_{s}^{2}-M_{\varphi}^{2}}{2 m_{i}}, E_{a}^{i}=\sqrt{p^{2}+m_{s}^{2}}+\frac{m_{i}^{2}-m_{s}^{2}+M_{\varphi}^{2}}{2 m_{i}},
$$

where $m_{i}$ is the mass of the $i$-th active neutrino, $M_{\varphi}$ is the mass of the pseudoscalar $\varphi$ and $m_{s}$ is the mass of the sterile neutrino. Since $m_{i} \ll m_{s}, M_{\varphi}$, from eq. (2) it follows that the resonance condition can be satisfied for positive energies if $m_{s}>M_{\varphi}$.

\section{CONSTRAINTS}

The proposed model could be subject to different constraints. We examine in detail the constraints on the secret interactions parameter space, coming both from laboratory experiments and from cosmological and astrophysical observations.

In the simple extension of the Standard Model under consideration, we introduce two new species of matter: the scalar field $\varphi$ and the sterile neutrino $v_{s}$. Our model is then parametrized by the set

$$
\left(\lambda_{\alpha}, M_{\varphi}, m_{s}\right) .
$$

The results of this analysis suggest a region of interest in the parameters $10 \mathrm{MeV}<m_{s}, M_{\varphi}<1$ $\mathrm{GeV}$.

- Laboratory bounds: Mesons can decay leptonically as $M \rightarrow v_{\ell} \ell$, where $M$ represents a meson $\left(\pi^{+}, K^{+}, D^{+}\right)$and $\ell=e, \mu, \tau$ depending on the meson. The interaction given in eq. (1) opens new leptonic 3 and 4 body decay channels: $M \rightarrow v_{s} \ell \varphi$ and $M \rightarrow v_{s} \ell \bar{v}_{\ell^{\prime}} v_{s}$. In this regard, we would like to remark that our assumption of small active-sterile mixing angle is crucial, since it prevents the appearance of new decay channels such as $M \rightarrow v_{\ell^{\prime}} \ell \varphi$. Concerning the process $M \rightarrow v_{s} \ell \varphi$, it becomes possible only if the corresponding $\lambda_{\ell} \neq 0$; 


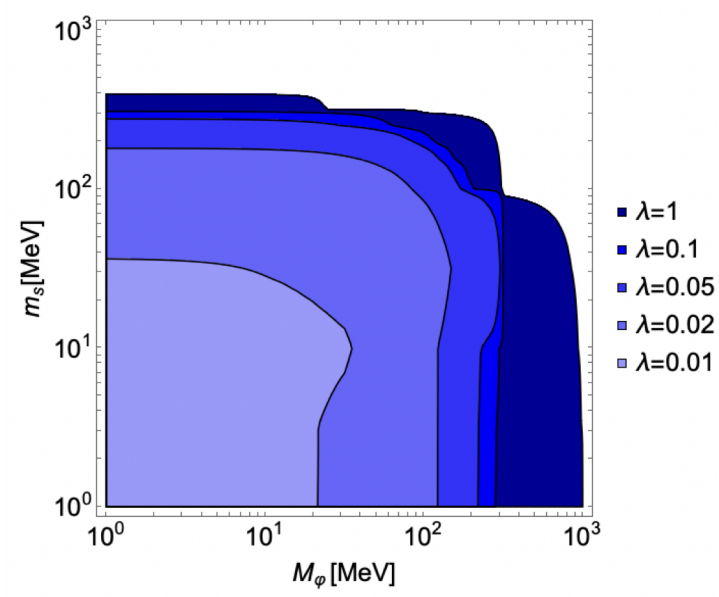

Figure 2: Exclusion contours in the $M_{\varphi}-m_{s}$ plane for different values of the coupling $\lambda=\lambda_{e}=\lambda_{\mu}=\lambda_{\tau}$, for the choice of equal flavor coupling: the region below the contours is excluded.

moreover, it is kinematically allowed only if $m_{s}+M_{\varphi} \lesssim m_{M}-m_{\ell}$, where $m_{M}$ is the mass of the decaying meson and $m_{\ell}$ the mass of charged lepton $\ell$. A lower limit on $m_{s}+M_{\varphi}$ arises from Big Bang Nucleosynthesis as discussed below. In the case of the four-body decay, $M \rightarrow v_{s} \ell \bar{v}_{\ell^{\prime}} v_{s}$, the kinematics only constrains the mass of the sterile neutrino. In particular, the decay is kinematically allowed if $2 m_{s} \lesssim m_{M}-m_{\ell}$ If $\lambda<1$, the rate for four-body decay will be smaller by a factor of $\lambda^{2}$ compared to the three-body decay ${ }^{1}$. We therefore expect that the four-body decay can relevantly change the exclusions in the parameter space only if $\lambda=1$.

Considering as example the Kaon decay into the muon channel, both the processes $K^{+} \rightarrow$ $\mu \varphi v_{s}$ and $K^{+} \rightarrow \mu v_{s} v_{s} \bar{v}_{\ell}^{\prime}$ should be observed as $K \rightarrow \mu+$ missing energy. We use then the closer Kaon decay process reported into PDG [7], namely $K \rightarrow \mu \nu \bar{v} v$, to constrain our processes imposing that the branching ratio to this channels should be smaller than $2.4 \times 10^{-6}$ [7]. In Fig. 2 we consider the case $\lambda_{e}=\lambda_{\mu}=\lambda_{\tau}=\lambda$ and we show the region excluded by Kaon decay in the $M_{\varphi}-m_{s}$ plane for various values of the coupling. We observe that for $\lambda \geq 0.01$ and $\left(m_{s}\right.$ or $\left.M_{\varphi}\right) \gtrsim 30 \mathrm{MeV}$, then the correction to Kaon decay is within the experimental bound. Concerning the four-body decay channel, this produces a bump in the right part of the exclusion contours, constraining $m_{s}$, and is relevant only for $\lambda=1$.

The case of only $\lambda_{\tau} \neq 0$ is unconstrained from meson physics and even for value of $\lambda_{\tau} \sim O(1)$ the only relevant bound in the $M_{\varphi}-m_{s}$ plane comes from Big Bang Nucleosynthesis as we will see below.

- Cosmological bounds: A first constraint comes from the Big Bang Nucleosynthesis (BBN) epoch and specifically from the requirement that there are no extra relativistic species at the moment of the BBN. This naturally happens if the new introduced species $\varphi$ and $v_{s}$ are non relativistic and in thermal equilibrium before and during BBN. Indeed, their distributions

${ }^{1}$ By $\lambda$ we mean the larger of the three couplings $\lambda_{\alpha}$. 
will be then Boltzmann suppressed by a factor of $e^{-m / T_{\mathrm{BBN}}}$, where $T_{\mathrm{BBN}} \simeq 1 \mathrm{MeV}$, and will not count as extra degree of freedom. Another constraint comes from the requirement that the active neutrinos should remain free-streaming (not collisional) at the time of the formation of the Cosmic Microwave Background ( $\mathrm{CMB}$ ) at temperature of the Universe around $\mathrm{eV}$. Limiting ourselves to an order of magnitude estimate of the rates of the relevant processes involving active neutrinos as a result of new interactions, we explore which portion of the parameter space is effectively constrained. Using an effective coupling $\lambda$, chosen as the largest coupling between the three flavours, the cosmological limits are satisfied for $m_{s} \gtrsim 10 \mathrm{MeV}$ and $M_{\varphi} \gtrsim 10 \mathrm{MeV}$.

- Astrophysical bounds: Supernovae neutrinos with energies of order of tens or hundreds of $\mathrm{MeV}$ can produce non relativistic sterile neutrinos which could escape the supernova giving rise to an observable energy loss. Computing the mean free path of the sterile neutrino inside the core and considering the threshold luminosity of the supernova SN 1987A, we have numerically verified that he model we consider is not constrained by supernova data for $m_{s}$ and $M$ larger than $10 \mathrm{MeV}$.

\section{NEUTRINO FLUXES AND TRANSPORT EQUATION}

Active-sterile neutrino interaction can be relevant at very different energy scales depending on the mass of the scalar mediator $\varphi$ : the energy scale at which the process of absorption over neutrinos from the Cosmic Neutrino Background (CNB) happens resonantly at energies around $M_{\varphi}^{2} / m_{\alpha}$. For an active neutrino mass of $0.1 \mathrm{eV}$, we find that this energy scale can range from $\mathrm{PeV}$ to energies of order $10^{4} \mathrm{PeV}$ in the selected parameter space. Close to the PeV scale, the dominant source of neutrinos is expected to be constituted by galactic and extragalactic astrophysical sources, such as Active Galactic Nuclei (AGN) and Gamma Ray Bursts (GRB). The details of the emitted neutrino spectra are sensitive to the physics of the sources. However, a good fit to the observed IceCube data in the region below the PeV is represented by a simple PL spectrum . Therefore, in this range of energy, we discuss the effect of the new interaction on a PL spectrum with parameters obtained by the fit to the IceCube data given in [8]. At higher energies, from $100 \mathrm{PeV}$, there are no experimental data on the neutrino flux. It is expected that a dominant source of neutrinos should have cosmogenic origin for which we use as a reference the spectrum proposed in [9] which constitutes a lower bound for the cosmogenic neutrino spectrum.

Because of the secret interactions, active neutrinos can collide with active neutrinos from the CNB, producing sterile neutrinos and thereby causing a depletion of the flux observable at Earth. The transport equation for active neutrinos is in principle coupled to the transport equation for sterile neutrinos, since the secret interactions produce sterile neutrinos which can in turn collide with other CNB neutrinos to regenerate part of the original flux. The form of these equations has been given in [1], and we reproduce it here for the generalized multiflavor case. We define with $\Phi_{i}(z, E)$ the flux of active neutrinos in the $i$ th $(i=1,2,3)$ mass eigenstate per unit energy interval per unit solid angle at a redshift $z$, while $\Phi_{s}(z, E)$ denotes the flux of sterile neutrinos, where in absence of mixing the sterile mass eigenstate is indicated with $s$. The flux at Earth is connected with the flux at generic redshift by the relation $\frac{d \phi_{\nu}}{d E d \Omega}=\Phi(0, E)$. We write separate equations for the 
mass eigenstates because, as discussed in [1], the propagation is diagonal in the mass eigenstates, given the path between collisions much larger than the oscillation lengths. In other words, due to the very fast oscillations caused by mixing, in between two collisions, a neutrino decoheres to mass eigenstates.

With negligible mixing between the active and the sterile neutrinos, we have no interest in the sterile flux at Earth, so the transport equation for active neutrinos take the form:

$$
\begin{aligned}
H(z)(1+z) & \left(\frac{\partial \Phi_{i}(z, E)}{\partial z}+\frac{\partial \Phi_{i}(z, E)}{\partial E} \frac{E}{1+z}\right)=n(z) \sigma_{i} \Phi_{i}(z, E) \\
& -\int d E^{\prime} \Phi_{s}\left(z, E^{\prime}\right) \frac{d \sigma_{s a}}{d E}\left(E^{\prime} \rightarrow E\right) n(z)-\rho(z)(1+z) f(E) \xi_{i},
\end{aligned}
$$

where $f(E)$ is the neutrino spectrum produced and $\xi_{i}$ the fraction of neutrinos produced at the source in the $i$ th mass eigenstate, produced at the source. $\sigma_{i}$ denotes the cross section for the collision of an $i$ th mass eigenstate with a CNB neutrino. Correspondingly, $\frac{d \sigma_{\alpha \beta}}{d E}\left(E^{\prime} \rightarrow E\right)$ is the cross section for the production of a $\beta$ neutrino with energy $E$ after the collision of a $\alpha$ neutrino with energy $E^{\prime}$ with a CNB neutrino. A simplification to numerical deal with this equation is to neglect the regeneration term representing the replenishment of the active flux by the collisions of daughter sterile neutrino with a cosmic neutrino (term $\propto \frac{d \sigma_{s a}}{d E}\left(E^{\prime} \rightarrow E\right)$ ). Indeed, adopting a perturbative approach, we found that both cosmogenic and astrophysical fluxes are practically unaffected by regeneration.

\section{OBSERVABLE DEPLETIONS}

We are ready now to discuss the results we obtained, namely the possible absorption features for astrophysical neutrinos and for cosmogenic ones which could be observable in present and future experiments, as consequence of secret active sterile neutrino interaction. Starting with an astrophysical power law flux in the range below $100 \mathrm{PeV}$, in the left panel of Fig.3 we show the astrophysical all flavor neutrino power law spectra in absence of SI, black continue curve, and in two cases of secret interaction corresponding two different coupling choices, namely $\lambda_{a f}$ (where $a f$ denotes all flavors), or $\lambda_{e}=\lambda_{\mu}=0$ and $\lambda_{\tau} \neq 0$, together with the IceCube HESE data. Remarkably, In the case in which the mediator only couples to tau neutrinos, the constraints from mesons decay are irrelevant and we can also consider lower masses for $M_{\Phi}$. We can infer that this possibility is already testable using IceCube data while the first case is essentially undistinguishable from the power- law in the energy range probed by IceCube. For the case of flux of cosmogenic neutrino, produced by the interaction of cosmic rays with the CMB photons, and which are relevant at higher energies, the absorption effect is most important for higher masses of the sterile neutrino and of the scalar mediator. In this part of the parameter space, the constraints from mesons decay are substantially irrelevant, so there is no need to distinguish between differente curling cases. So in the right panel of Fig. 3 we show, for $\lambda_{a f}=1$, the expected cosmogenic in the case of pure protonic cosmic rays, in absence of secret interactions (continuous light green curve) and in presence of secret interactions for some benchmark values of the scalar mediator mass while the value of the sterile mass if fixed in all the cases. Larger masses for the mediator correspond to weaker absorption. 

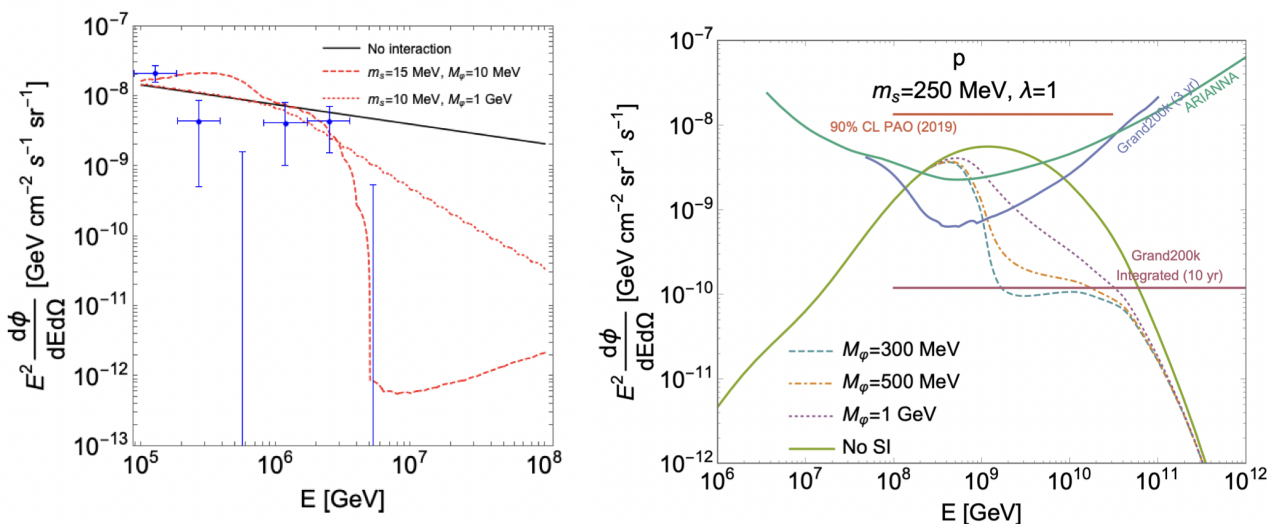

Figure 3: Left panel: astrophysical all flavor neutrino power law spectra: the thick line is the flux with no interaction, while the dotted line corresponds to the case of $\lambda_{a f}=1$ and the dashed one denotes the case $\lambda_{\tau}=1$. The experimental points are the IceCube HESE data. Right panel: effects on the cosmogenic spectrum expected in the case of proton cosmic rays, where the continuous green curve is the cosmogenic spectrum in the absence of secret interactions while the dashed, dot-dashed and dotted lines are the spectrum for different combinations secret interactions. The sensitivity of the GRAND experiment, the 90\% C.L. of PAO, the integrated sensitivity of GRAND after 10 years of data and the sensitivity of the ARIANNA experiment are also shown.

\section{Acknowledgments}

This work was supported by the Italian grant 2017W4HA7S "NAT-NET: Neutrino and Astroparticle Theory Network" (PRIN 2017) funded by the Italian Ministero dell'Istruzione, dell'Universitá e della Ricerca (MIUR), and Iniziativa Specifica TAsP of INFN.

\section{References}

[1] D. F. G. Fiorillo, G. Miele, S. Morisi and N. Saviano, Phys. Rev. D 101 (2020) no.8, 083024 doi:10.1103/PhysRevD.101.083024 [arXiv:2002.10125 [hep-ph]].

[2] D. F. G. Fiorillo, S. Morisi, G. Miele and N. Saviano, Phys. Rev. D 102 (2020) no.8, 083014 doi:10.1103/PhysRevD.102.083014 [arXiv:2007.07866 [hep-ph]].

[3] X. Chu, B. Dasgupta, M. Dentler, J. Kopp and N. Saviano, JCAP 11 (2018), 049 doi:10.1088/1475-7516/2018/11/049 [arXiv:1806.10629 [hep-ph]].

[4] F. Forastieri, M. Lattanzi, G. Mangano, A. Mirizzi, P. Natoli and N. Saviano, JCAP 07 (2017), 038 doi:10.1088/1475-7516/2017/07/038 [arXiv:1704.00626 [astro-ph.CO]].

[5] A. Mirizzi, G. Mangano, O. Pisanti and N. Saviano, Phys. Rev. D 91 (2015) no.2, 025019 doi:10.1103/PhysRevD.91.025019 [arXiv:1410.1385 [hep-ph]].

[6] N. Saviano, O. Pisanti, G. Mangano and A. Mirizzi, Phys. Rev. D 90 (2014) no.11, 113009 doi:10.1103/PhysRevD.90.113009 [arXiv:1409.1680 [astro-ph.CO]]. 
[7] M. Tanabashi et al. [Particle Data Group], Phys. Rev. D 98 (2018) no.3, 030001 doi:10.1103/PhysRevD.98.030001

[8] D. R. Williams [IceCube], Int. J. Mod. Phys. Conf. Ser. 46 (2018), 1860048 doi:10.1142/S2010194518600480 [arXiv:1909.05173 [astro-ph.HE]].

[9] M. Ahlers and F. Halzen, Phys. Rev. D 86 (2012), 083010 doi:10.1103/PhysRevD.86.083010 [arXiv:1208.4181 [astro-ph.HE]]. 